\title{
Colorectal Cancer Screening in Ghana: Physicians' Practices and Perceived Barriers
}

\author{
Alisha Lussiez ${ }^{1}$ (1) - Shukri H. A. Dualeh ${ }^{1}$ Charles K. Dally ${ }^{2,3}$ - Baafuor K. Opoku ${ }^{3,4}$ • \\ Krishnan Raghavendran ${ }^{1,5} \cdot$ Francis Aitpillah $^{2} \cdot$ Edward Boateng $^{2} \cdot$ Dominic Darkwah $^{2}$. \\ Kofi Christian Gyasi-Sarpong ${ }^{2,3} \cdot$ Joseph C. Kolars $^{6} \cdot$ Gifty Kwakye $^{1,5,7}$
}

Accepted: 11 October 2020/Published online: 3 November 2020

(C) Société Internationale de Chirurgie 2020

\begin{abstract}
Introduction Ghana has seen a rise in the incidence of colorectal cancer (CRC) over the past decade. In 2011, the Ghana National Cancer Steering Committee created a guideline recommending fecal occult blood testing (FOBT) for CRC screening in individuals over the age of 50. There is limited data available on current Ghanaian CRC screening trends and adherence to the established guidelines.

Methods We conducted a survey of 39 physicians working at the Komfo Anokye Teaching Hospital in Kumasi, Ghana. The survey evaluates physician knowledge, practice patterns, and perceived personal-, patient- and systemlevel barriers pertaining to CRC screening.

Results Almost $10 \%$ of physicians would not recommend colorectal cancer screening for asymptomatic, average risk patients who met the age inclusion criteria set forth in the national guidelines. Only 1 physician would recommend FOBT as an initial screening test for CRC. The top reasons for not recommending CRC screening with FOBT were the lack of equipment/facilities for the test $(28.1 \%)$ and lack of training (18.8\%). The two most commonly identified barriers to screening identified by $>85 \%$ of physicians, were lack of awareness of screening/not perceiving colorectal cancer as a serious health threat (patient-level) and high screening costs/lack of insurance coverage (system-level). Conclusion Despite creation of national guidelines for CRC screening, there has been low uptake and implementation. This is due to several barriers at the physician-, patient- and system-levels including lack of resources and physician training to follow-up on positive screening results, limited monetary support and substantial gaps in knowledge at the patient level.
\end{abstract}

Gifty Kwakye

gkwakye@umich.edu

Alisha Lussiez

alussiez@med.umich.edu

Shukri H. A. Dualeh

shukrid@med.umich.edu

Charles K. Dally

dallykc@yahoo.com

Baafuor K. Opoku

baafuoropoku@yahoo.com

Krishnan Raghavendran

kraghave@med.umich.edu
Francis Aitpillah

fraitp@yahoo.com

Edward Boateng

eaboateng@hotmail.co.uk

Dominic Darkwah

stakigh@msn.com

Kofi Christian Gyasi-Sarpong

gaysek@yahoo.com

Joseph C. Kolars

jckolars@med.umich.edu

Department of Surgery, University of Michigan, 1500 East Medical Center Dr, Ann Arbor, MI 48109, USA 


\section{Introduction}

Colorectal cancer is the second and the third most common cancer among men and women worldwide, respectively [1]. Ghana has seen a rise in the incidence of colorectal cancer over the past decade [2-4]. Specifically, the number of new cases has increased by 8 -fold from an average of 4.1 new cases per 100,000 per year in 1960s to an average of 32.6 new cases per 100,000 per year in 2017 with a noted high incidence in younger people and delayed presentation of the disease [4]. This, in part, has been attributed to the increased adoption of Western lifestyles including diet, physical inactivity, improved life expectancy (from 57 years in 2000 to 63 years in 2016) [4] and establishment of screening guidelines. In 2011, the Ghana National Cancer Steering Committee identified colorectal cancer as one of its targets for improvement efforts [3]. Using limited available data, the Committee determined that there was not enough demand to support instituting endoscopic screening nationwide. Instead, a recommendation to use fecal occult blood testing (FOBT) as an initial screening tool for patients aged 50-70 years old, followed by endoscopic evaluation for those with positive results, was made [3].

Implementation of an effective colorectal cancer screening program has the potential to make a significant impact on the burden of disease as many colorectal cancers are curable if detected early and preventable if excised at the pre-malignant stage [5, 6]. Unfortunately, nationwide cancer screening efforts remain a significant challenge in Ghana, even with more prevalent diseases such as prostate, breast and cervical cancers $[3,7,8]$. There is limited data available on current Ghanaian colorectal cancer screening practices and no data regarding barriers to screening despite the establishment of national guidelines. Furthermore, the public health impact and adherence to the guidelines set forth by the Ghana National Cancer Steering Committee remains unclear.

In this study, we sought to better understand the current colorectal cancer screening practices of Ghanaian physicians across several different specialties. We hypothesized that, similar to breast and cervical cancer screening, there exist many physician-, patient- and system-level barriers that prevent widespread colorectal cancer screening and uptake of the national guidelines [7, 8]. Identification of these barriers is necessary to improve screening and ultimately increase early detection, prevention and cure of colorectal cancers.

\section{Materials and methods}

\section{Study design and setting}

We conducted a survey of physicians working at the Komfo Anokye Teaching Hospital (KATH) in Kumasi, Ghana. KATH is the second largest tertiary facility in Ghana, having 1,200 beds and 13 clinical directorates consisting of anesthesia and intensive care, child health, eye/ear/nose/throat (EENT), emergency medicine, family medicine, laboratory services, medicine, obstetrics and gynecology, oral health, surgery, trauma and orthopedics, and radiology. The hospital receives referrals from 12 of the 16 regions of Ghana, in addition to neighboring countries Burkina Faso and Cote D'Ivoire [9]. KATH plays an extensive and important role in healthcare delivery in Ghana and has demonstrated institutional investment to understand colorectal cancer screening patterns and disease volumes, making it an excellent site to conduct this study.

\section{Survey instrument}

The survey tool was adapted from a validated nationally used US Survey of Colorectal Cancer Screening Practices [10], sponsored by the US National Cancer Institute, Center for Disease Control and Prevention, and Health Care Financing Administration. The original survey was designed to accommodate a wide range of physicians and practice settings (including rural), which made it suitable for the purposes of this study. Physician knowledge,

6 University of Michigan Medical School, Ann Arbor, MI, USA

7 University of Michigan, TAUBMAN CENTER, Floor 2 Reception C, 1500 E Medical Center Dr SPC 5331, Ann Arbor, MI 48109-5331, USA 
attitudes and practice patterns pertaining to colorectal cancer screening are ascertained by exploring the following categories:

1. Demographics and Practice characteristics.

2. General cancer screening beliefs and practices.

(a) Effectiveness/ineffectiveness of screening procedures —including Pap smear, mammography, and prostate-specific antigen-in reducing mortality in average-risk patients aged 50 years and older.

(b) Choice of colorectal screening modality for average-risk patients.

3. Attitudes towards and perceived barriers to colorectal cancer screening.

(a) Physician's personal barriers to recommending colorectal cancer screening.

(b) Physician perceived patient- and system-related barriers to colorectal cancer screening.

To ensure relevance of survey questions to the Ghanaian physician population, questions on insurance, practice models (e.g., employee of physician owned practice or group model HMO), and specialty guidelines (e.g., American Cancer Society or U.S Preventive Services Task Force), for instance, were excluded. The final adapted survey was reviewed by two physicians at KATH to ensure appropriateness and familiarity of terms used ("Appendix"). Approval for the study was obtained from the Institutional Review Board at KATH as the primary site and additionally at the University of Michigan for secondary analyses.

\section{Survey distribution}

Our target population were physicians in clinical directorates that were involved in prevention, diagnosis and management of colorectal cancers. Directorates included were family medicine, gastroenterology, internal medicine, oncology, laboratory services/pathology and surgery. Endoscopic procedures at KATH are performed by both general surgery and gastroenterology due to significant national shortage of trained personnel. Of the 14 practicing faculty gastroenterologists in Ghana, KATH has only one serving its population [11]. Physicians in these directorates over the age of 18 were eligible to participate if they had at least one year of practice experience. The surveys were initially distributed in person but after the onset of the COVID-19 pandemic, an electronic system was utilized instead using Qualtrics survey platform [12]. Informed consent to participate in the study was implied by completing and returning the survey questionnaire.

\section{Statistical analysis}

Data were qualitatively analyzed using Qualtrics and Excel. Frequency distributions for categorical variables were examined to identify patterns and trends among the respondents. Not all participants answered every question. If a participant did not respond to a question, these data were not included in the analysis allowing percentages reported to represent a portion of a whole set (100\%).

\section{Results}

\section{Demographics and practice characteristics}

Either hardcopy or electronic surveys were distributed to 105 physicians between the months of February and May 2020. Thirty-nine physicians completed the survey with a $37 \%$ response rate; $92 \%$ of participants were male. Only 6 of the physicians who responded to the survey were between 51 and 70 years of age. The majority of surveyed physicians practiced in the field of general surgery $(41.7 \%)$ and held faculty positions $(69.4 \%)$. About half of participants (49\%) had been in practice for less than 10 years. The full breakdown of demographics can be seen in Table 1 .

\section{General cancer screening beliefs and practices}

\section{Effectiveness/Ineffectiveness of cancer screening procedures}

Participants' beliefs about the effectiveness of different cancer screening modalities are presented in Fig. 1. Cervical cancer screening using Pap smear was reported as being "very effective" by the largest proportion of physicians (82.6\%). Physicians were almost evenly split in regard to the effectiveness of mammography, rating it as either very or somewhat effective at screening for breast cancer. In regard to prostate-specific antigen (PSA), 43\% thought it was a very effective screening tool, 53\% somewhat effective and 3\% not effective for diagnosing prostate cancer. When presented with the various screening modalities for colorectal cancer, respondents found colonoscopy and flexible sigmoidoscopy to be very effective (66.7\% and 55.5\%, respectively). The distribution for fecal occult blood testing (FOBT) was $25 \%$ very effective, $71.4 \%$ somewhat effective and $3.6 \%$ not effective.

Choice of colorectal screening modality for average-risk patients

For an asymptomatic, average-risk patient, a majority of participants $(40 \%)$ were likely to initially recommend 
Table 1 Demographics

\begin{tabular}{lc}
\hline Variable & $N(\%)$ \\
\hline Gender & \\
Male & $35(92.1 \%)$ \\
Female & $3(7.9 \%)$ \\
Age (years) & \\
$<31$ & $5(13.5 \%)$ \\
$31-50$ & $26(70.3 \%)$ \\
$51-70$ & $6(16.2 \%)$ \\
Specialty & \\
General surgery & $15(41.7 \%)$ \\
Family medicine & $3(8.3 \%)$ \\
Oncology & $4(11.1 \%)$ \\
Other surgical sub-specialty & $11(30.7 \%$ \\
Pathology & $3(8.3 \%)$ \\
Years in practice & \\
$\leq 5$ & $9(23.7 \%)$ \\
$6-10$ & $10(26.3 \%)$ \\
$11-15$ & $5(13.1 \%)$ \\
$16-20$ & $11(28.9 \%)$ \\
$>20$ & $3(7.9 \%)$ \\
Level of training & \\
Faculty/attending/consultant & $25(69.4 \%)$ \\
Resident & $11(30.6 \%)$ \\
\hline
\end{tabular}

screening with colonoscopy alone; $26.7 \%$ would recommend FOBT in combination with flexible sigmoidoscopy $(26.7 \%)$. Only one physician recommended FOBT alone as an initial screening test. And $9.6 \%$ of physicians would not recommend colorectal cancer screening with any modality for asymptomatic, average-risk patients (Table 2). No significant differences were identified when screening test recommendations were stratified by specialty. When asked which screening modality was recommended after patients presented with a positive FOBT, $66.7 \%$ recommended colonoscopy and $33.3 \%$ recommended flexible sigmoidoscopy (Table 2).

\section{Attitudes towards and perceived barriers to colorectal cancer screening}

Physician's personal barriers to recommending colorectal cancer screening

The top three reasons for not recommending or not always performing colorectal cancer screening with FOBT in asymptomatic, average-risk patients (Fig. 2) were: (1) lacking the equipment/facilities for the procedure $(28.1 \%)$, (2) lacking the training in the conduct of the test $(18.8 \%)$ and (3) poor patient compliance (18.8\%). Some of the "Other" reasons cited included: "most of my patients are symptomatic" and "not in my routine line of practice."

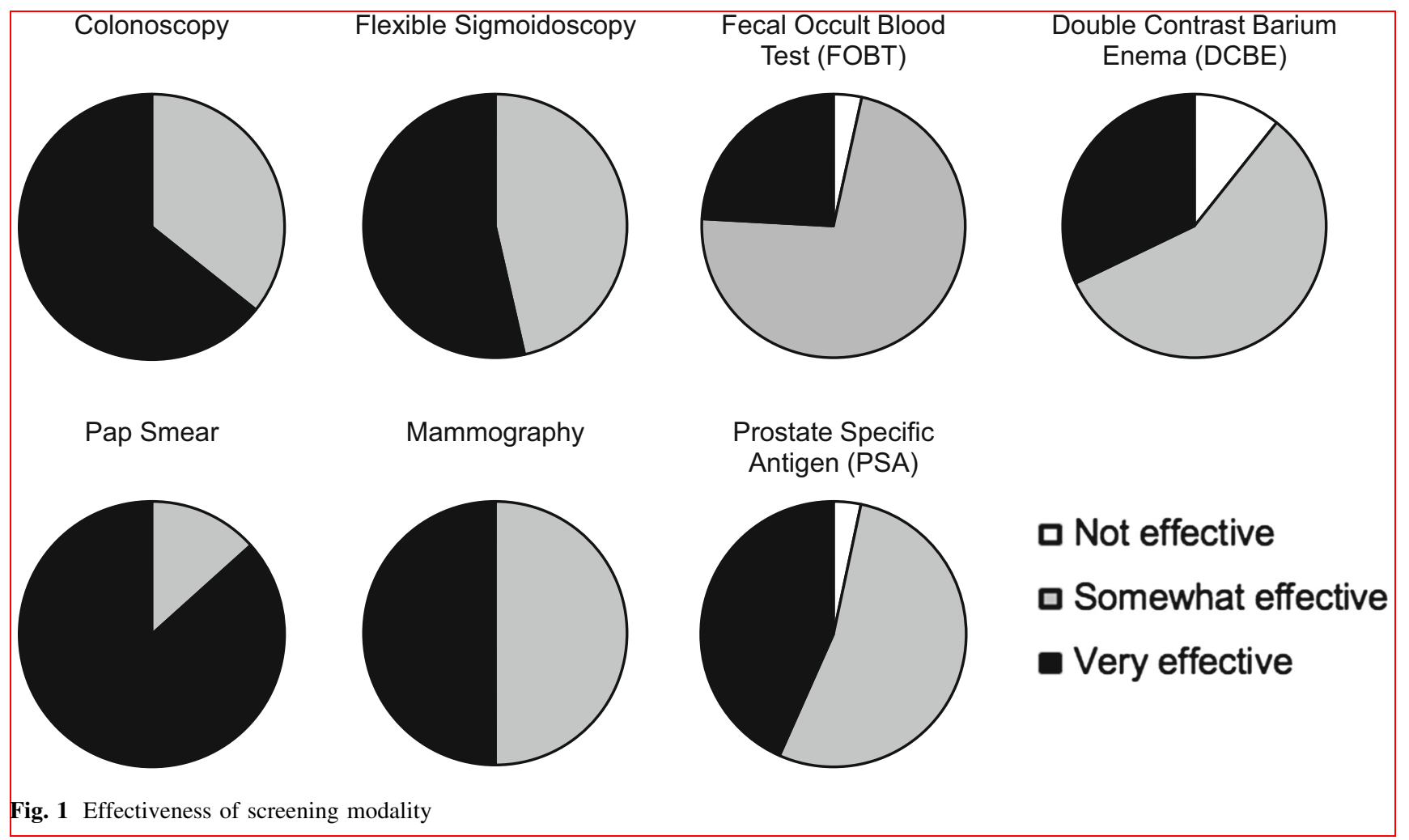


Table 2 Breakdown of recommended CRC screening modality for asymptomatic, average-risk patients

\begin{tabular}{lll}
\hline Screening modality & $\begin{array}{l}\text { Without } \\
\text { previous }\end{array}$ & $\begin{array}{l}\text { After } \\
\text { positive }\end{array}$ \\
& $\begin{array}{l}\text { FOBT } \\
\text { FOBT } \\
N(\%)\end{array}$ \\
\hline FOBT alone & $1(3.2 \%)$ & \\
FOBT OR flexible sigmoidoscopy & $2(6.5 \%)$ & \\
FOBT AND flexible sigmoidoscopy & $9(29.0 \%)$ & \\
Flexible sigmoidoscopy alone & $2(6.5 \%)$ & $9(33.3 \%)$ \\
Colonoscopy & $12(38.7 \%)$ & $18(66.7 \%)$ \\
Double contrast barium enema & $1(3.2 \%)$ & \\
I do not recommend CRC screening & $3(9.6 \%)$ & \\
Other & $1(3.2 \%)$ & \\
\hline
\end{tabular}

None of the physicians between 51 and 70 years of age had undergone colorectal cancer screening. Of those younger than 50 years, $12 \%$ had been screened with either FOBT, flexible sigmoidoscopy or colonoscopy.

\section{Physician perceived patient- and system-related barriers to colorectal cancer screening}

Figure 3 shows the breakdown of identified physician perceived patient- and system-related barriers to colorectal cancer screening. Major barriers to screening identified were: patients' lack of awareness of screening/not perceiving colorectal cancer as a serious health threat $(86.2 \%)$, high screening costs/lack of insurance coverage $(85.7 \%)$, primary care physician (PCP) doesn't actively recommend screening $(65.6 \%)$, shortage of trained providers to conduct follow-up invasive procedures $(60.7 \%)$ and shortage of trained providers for other screening modalities besides FOBT $(58.6 \%) ; 78.6 \%$ of physicians did not identify patients "believing screening to be ineffective" as a barrier.

\section{Discussion}

In this study, we report that colorectal cancer screening practices vary widely among Ghanaian physicians, and for the most part, are quite discordant with the national guidelines. Reasons for this are multi-factorial and include physician-, patient- and system-level barriers to colorectal cancer screening. Of these barriers, system-level barriers were identified to be the greatest impediment. This has significant implications on (1) recommendations made to patients, with almost $10 \%$ of physicians reporting that they would not recommend or order colorectal cancer screening for average risk, asymptomatic patients, who meet the established age criteria and (2) physician personal adherence, with none of the physicians aged 51-70 in our study having ever undergone colorectal cancer screening.

At the physician-level, the most commonly identified reasons for not recommending colorectal cancer screening were a lack of equipment/facilities, inadequate training on how to perform screening procedures, as well as poor patient compliance. Furthermore, physicians cited a lack of training on how to perform screening beyond FOBT $(86.2 \%)$ and the inability to perform follow-ups requiring invasive procedures $(94.3 \%)$ as barriers to colorectal cancer screening. This suggests physicians may find screening with FOBT futile as they are not able to provide patients

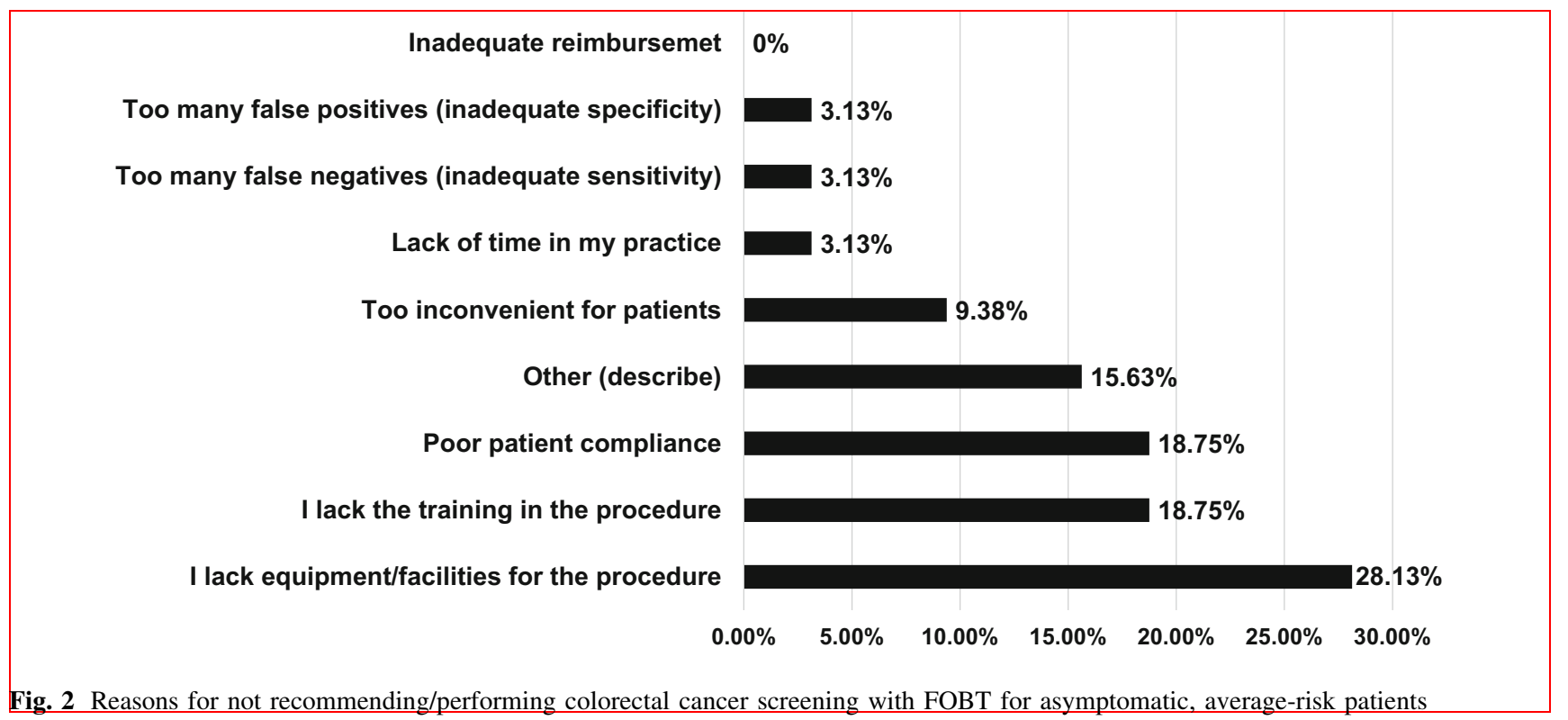




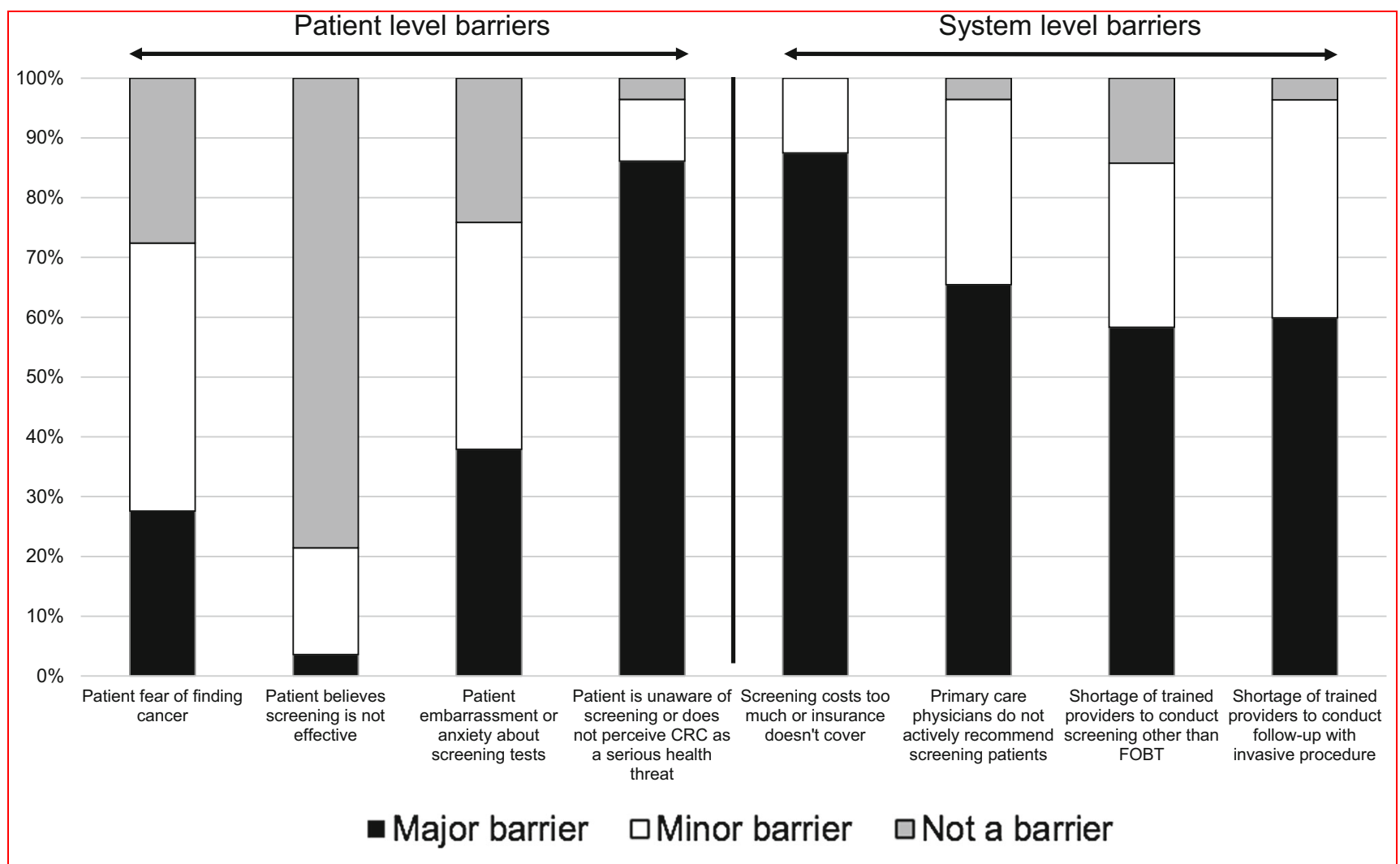

Fig. 3 Barriers to colorectal cancer screening

with the indicated next steps in diagnosis and treatment. In the study by Dakubo et al. [13] complete colonoscopy rates were only $30.4 \%$ due to inadequate endoscopic training, incomplete bowel prep and patient discomfort from inadequate sedation. Unless endoscopic training and resource availability are made a priority, rates of complete colonoscopies are unlikely to increase and many physicians will continue to defer screening with FOBT.

Even though the majority of physicians felt their patients believed screening was effective, they did note that it was unlikely patients were aware of screening recommendations or perceived colorectal cancer as a serious health threat. This combination of findings suggests the existence of a large gap in education regarding the lifelong medical, financial, psychological and social implications of a colorectal cancer diagnosis as well as the benefits of early detection and indications for screening. Patients may be unaware that early detection and treatment of colorectal cancers can drastically reduce the mortality related to these diseases [3]. Furthermore, patients likely do not recognize the growing need for colorectal cancer screening given the increasing incidence of colorectal cancers due to patient factors such as the expanding adoption of the Western diet and lifestyle [4].
This lack of medical knowledge is likely accompanied by several non-physical barriers to compliance with colorectal cancer screening in Ghana. Although this has not yet been explicitly studied, we can draw on the similarities of behaviors and beliefs surrounding cervical cancer, another cancer of a sensitive region. The World Health Organization (WHO) has suggested that major barriers to the control of cervical cancer in developing countries such as Ghana, include women's attitudes, misconceptions, and beliefs about cervical cancer. This is supported by the study from Kuffour et al. "Assessment of psychological barriers to cervical cancer screening among women in Kumasi, Ghana using a mixed methods approach.” In this study, the authors uncover several misconceptions about cervical cancer including its cause and risk factors. For example, only one participant knew that cervical cancer is caused by the human papilloma virus (HPV). Several participants thought that having abortions, inserting herbs or other substances into the vagina or exposing the vagina to chemicals caused cervical cancer [14]. These nonphysical barriers to screening and misconceptions regarding colorectal cancers also likely exist. Further studies to understand the exact beliefs and level of knowledge held by the Ghanaian population are needed. 
At the system-level, the financial cost of screening was cited as the greatest barrier. Despite publication of guidelines created by the Ghana National Steering Committee, colorectal cancer screening has not received significant national financial support. The cost of FOBT ranges from 30 to 50 Ghana cedi which places a significant financial burden on most patients as the daily minimum wage is 11.92 Ghana cedi (equivalent to 2.16 US dollars) [15]. Notably, it is not covered by the National Health Insurance Scheme (NHIS) unlike cervical and breast cancer [16]. As such, patients have to pay out of pocket for screening tests, laboratory analysis and any subsequent intervention needed. This creates an unfair dilemma for patients and their families, forcing them to choose between competing financial priorities. If any actual progress is to be made in countering the rising rates of colorectal cancer in Ghana, it will need sustained commitment and financial investment from the government. Studies that provide data on the true incidence of colorectal cancer and highlight both the individual and societal costs, will be crucial in motivating stakeholders to support colorectal cancer prevention on a national scale.

Limitations of our study include its conduct at a single institution along with a low (37\%) response rate, both of which are factors that might limit the generalizability of this data. The low response rate may reflect overwhelming clinical duties and limited access to reliable internet brought on by the COVID pandemic. Separately, we were unable to identify significant differences in practice habits between older and younger physicians since age data were collected in wide ranges. Given the evolving colorectal surgery training, in future studies we will breakdown age categories into smaller groups to allow for more detailed analysis. Lastly, our study did not address the degree of physician-awareness regarding the guidelines set forth by the Ghana National Cancer Steering Committee, but we plan to further investigate the above with semi-structured patient and physician interviews.

\section{Conclusion}

The incidence of colorectal cancers in Ghana is rising. Despite creation of national guidelines for colorectal cancer screening, there has been low uptake and implementation. This is due to several barriers at the physician-, patient- and system-levels including lack of resources and physician training to follow-up on positive screening results, limited monetary support to pay for screening tests and substantial gaps in knowledge at the patient level surrounding the lifelong medical, financial, psychological and social consequences of a colorectal cancer diagnosis.

Funding Dr. Lussiez is supported by the National Cancer Institute under award number 5T32CA009672-30.

\section{Compliance with ethical standards}

Conflict of interest The authors declare that they have no conflict of interest.

\section{Appendix}




\section{KATH CRC Awareness}

\section{Start of Block: Default Question Block}

\section{Q1 Survey of Colorectal Cancer Screening Practices Physician}

Questionnaire

In this survey, cancer

screening is defined as the routine, periodic use of a testing procedure intended to detect cancer or pre-cancerous lesions at an earlier stage than is possible through clinical detection or incidental discovery. Cancer screening is used in patients who display no signs or symptoms of possible cancer (i.e., pain, bleeding, palpable masses, etc.)

Page Break

Q3 Part 1. Provider Demographic data

Q4 How old are you?

less than 30 years $(1)$

$31-50$ years $(2)$

$51-70$ years $(3)$

older than 71 years (4)

Q5 Gender

Male (1)

Female (2)

Q6 Specialty or Directorate

Q7 Number of years in practice?
less than 5 years (1)
$6-10$ years (2)
$11-15$ years $(3)$
16- 20 years (4)
more than 21 years (5) 
Q8 During a typical week, approximately how many patients do you see?
75 or fewer (1)
$76-100(2)$
$101-125$
126 or more (4)

Q9 Approximately what percentage of your patients are 50 years of age or older?
Less than $25 \%$ (1)
$25-49 \%(2)$
$50-74 \%(3)$
$75-100 \%(4)$

Page Break

Q10 Part 2. Cancer Screening Beliefs and Practices This section includes questions about your experiences with cancer screening. Please respond based on how you actually practice even if this differs from how you would like to practice under ideal circumstances.

Q11 How effective or ineffective do you believe the following screening procedures are in reducing cancer mortality in average-risk patients aged 50 years and older? (SELECT ONE ANSWER ON EACH LINE)

\begin{tabular}{c|cc} 
Very Effective & $\begin{array}{c}\text { Somewhat } \\
\text { Effective (2) }\end{array}$
\end{tabular}$\quad$ Not Effective (3) Don't Know (4)

Pap Smear (1)

Mammography

Prostate Specific Antigen (PSA)

Fecal Occult Blood Test (FOBT) (4)

Flexible sigmoidoscopy (5)

Colonoscopy (6)

Double Contrast Barium Enema (DCBE) (7) 
Q12 In your practice, do you routinely order or perform the following cancer screening procedures for your asymptomatic, average-risk patients? (SELECT ONE ANSWER ON EACH LINE)

\begin{tabular}{c|cc} 
& Yes (1) No (2) \\
\hline Pap Smear (1) & 0 & \\
Mammography (2) & & \\
Prostate Specific Antigen \\
(PSA) (3)
\end{tabular}

Q14 Please fill in each of the following boxes. OK to write N/A if you either don't recommend the test routinely or don't know.

\begin{tabular}{c|c|c|c} 
& $\begin{array}{c}\text { Recommended } \\
\text { starting age? (1) }\end{array}$ & $\begin{array}{c}\text { Recommended } \\
\text { frequency of testing? }\end{array}$ & $\begin{array}{c}\text { At what age do you } \\
\text { recommend no } \\
\text { further testing? (3) }\end{array}$ \\
\hline Pap Smear (1) & & & \\
\hline Mammography (2) & & & \\
\hline Prostate Specific & & & \\
\hline Antigen (PSA) (3) & & & \\
\hline & & & \\
\hline & & & \\
\hline
\end{tabular}

Q15 Which test or test combination do you most often recommend to your asymptomatic, average-risk patients as a colorectal cancer screening strategy? (SELECT ONLY ONE ANSWER)

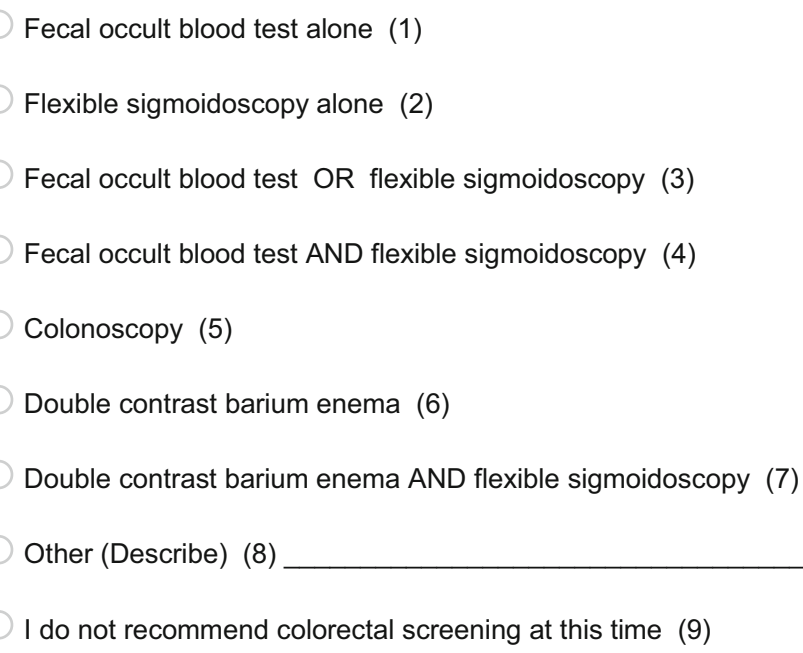


Q16 How many times over the past month have you personally ordered or performed, or supervised a nurse or trainee do a fecal occult blood test for colorectal cancer screening for one of your asymptomatic, average-risk patients?
(1)
$1-10(2)$
$11-20(3)$
$21-40(4)$
more than 40

Q17 Which of the following do you usually recommend to a healthy, average-risk patient as an initial follow-up step to a positive Fecal Occult Blood Test ( FOBT)? (SELECT ALL THAT

APPLY)

Repeat FOBT (1)

Flexible sigmoidoscopy (2)

Colonoscopy (3)

Double contrast barium enema (4)

Other (Describe) (5)

Q18 What are your reasons for not recommending or not always performing colorectal cancer screening with FOBT for asymptomatic, average-risk patients? (SELECT ALL THAT APPLY)

I lack equipment/facilities for the procedure (1)

I lack the training in the procedure (2)

Lack of time in my practice $(3$

Too many false negatives (inadequate sensitivity) (4)

Too many false positives (inadequate specificity) (5)

Too inconvenient for patients (6)

Poor patient compliance (7)

Inadequate reimbursement (8)

Other (Describe) (9) 
Q19 Over the past 2 years, has the volume of colorectal cancer screening procedures you order, perform or supervise:

Increased substantially (more than $20 \%$ per year) (1)

Increased somewhat (less than $20 \%$ per year) (2)

Stayed about the same (3)

Decreased (4)

Q20 Please comment on the current capacity of facilities and personnel in your community for performing these cancer screening procedures over the next 3 years. (SELECT ONE ANSWER ON EACH LINE)

$\begin{array}{cccc}\begin{array}{c}\text { More than } \\ \text { enough to meet } \\ \text { demand (1) }\end{array} & \begin{array}{c}\text { Just about right } \\ \text { to meet demand }\end{array} & \begin{array}{c}\text { Inadequate to } \\ \text { meet demand (3) }\end{array} & \text { Don't know (4) }\end{array}$

\section{Flexible} sigmoidoscopy

Colonoscopy (2)

Double contrast barium enema

(3)

Q21 Have you ever ordered, referred, or recommended a patient for genetic testing for a suspected inherited susceptibility to colorectal cancer based on a strong family history?

Yes, I have ordered (1)

Yes, I have referred or recommended (2)

No (3)

Q22 Have you personally ever been screened for colorectal cancer? (CHOOSE ALL THAT APPLY)

Yes, with Fecal occult blood test (FOBT) (1)

Yes, with flexible sigmoidoscopy (2)

Yes, with colonoscopy (3)

Yes, with Double contrast barium enema (4)

No, I have not been screened (5) 
Q23 In the Ghana today, most adults over the age of 50 are not screened for colorectal cancer. In your opinion, how important are each of the following as potential barriers to colorectal cancer screening? (SELECT ONE ANSWER ON EACH LINE)

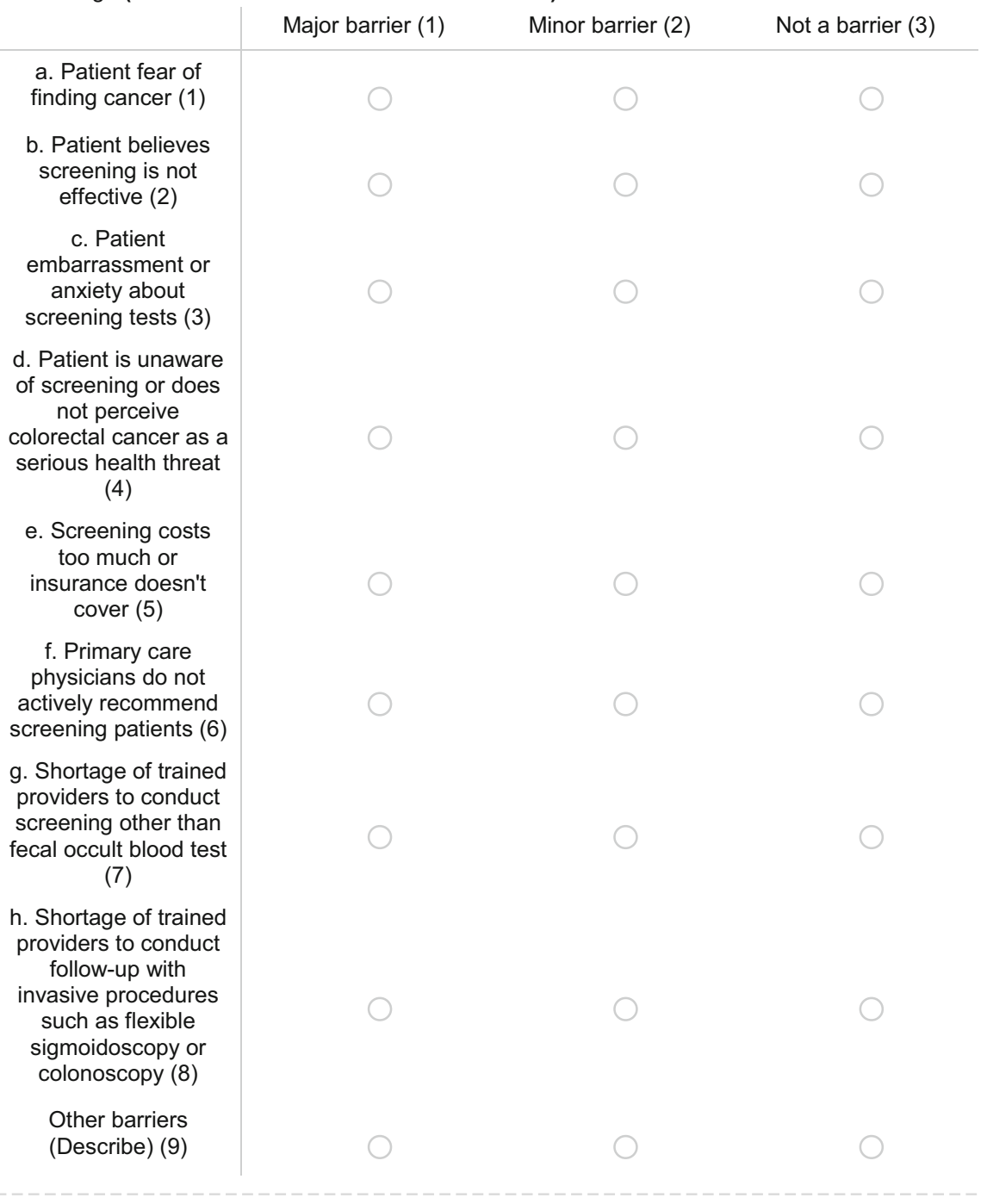

Q24 Is there anything else you would like to tell us about colorectal cancer screening in your practice or in general? understand the existing colorectal cancer screening patterns in your institution and challenges to providers. 


\section{References}

1. Globocan database (2012) Cancers fact sheets: colorectal cancer, pp 1-10. http://gco.iarc.fr/today

2. Calys-Tagoe BN, Yarney J, Kenu E, Owusu Amanhyia NAK, Enchill E, Obeng I (2014) Profile of cancer patients' seen at Korle $\mathrm{Bu}$ teaching hospital in Ghana (A cancer registry review). BMC Res Notes 7(1):1-6. https://doi.org/10.1186/1756-0500-7-577

3. MoH (2016) National Strategy for cancer. Minist Heal Ghana, pp 1-8

4. Yeboah FA, Yorke J, Obirikorang C et al (2017) Patterns and presentations of colorectal cancer at Komfo-Anokye teaching hospital Kumasi, Ghana. Pan Afr Med J 28:1-10. https://doi.org/ 10.11604/pamj.2017.28.121.12927

5. Survival Rates for Colorectal Cancer (2020) https://www.cancer. org/cancer/colon-rectal-cancer/detection-diagnosis-staging/survi val-rates.html. Accessed 1 July 2020

6. Tfaily MA, Naamani D, Kassir A et al (2019) Awareness of colorectal cancer and attitudes towards its screening guidelines in lebanon. Ann Glob Health 85(1):1-11. https://doi.org/10.5334/ aogh. 2437

7. Opoku SY, Benwell M, Yarney J (2012) Knowledge, attitudes, beliefs, behaviour and breast cancer screening practices in Ghana, West Africa. Pan Afr Med J 11:28. https://doi.org/10.11604/pamj. 2012.11.28.548

8. Ebu NI, Amissah-Essel S, Asiedu C, Akaba S, Pereko KA (2019) Impact of health education intervention on knowledge and perception of cervical cancer and screening for women in Ghana.
BMC Public Health 19(1):1-11. https://doi.org/10.1186/s12889019-7867-x

9. Komfo Anokye Teaching Hospital-About Us (2019). http://www. kathhsp.org/about-us/. Accessed 1 July 2020

10. Financing HC, Associates A. Survey of colorectal cancer screening practices primary care physician questionnaire. https:// healthcaredelivery.cancer.gov/crc_surveys/prim0520.pdf. Accessed 1 Feb 2020

11. MedPages. https://www.medpages.info/sf/index.php?page=lis ting \& servicecode $=168 \&$ countryid $=16 \&$ regioncode $=\&$ sub regioncode $=$. Accessed 1 July 2020

12. Qualtrics (2020.) https://www.qualtrics.com. Accessed 1 July 2020

13. Dakubo JCB, Kumoji R, Aaeder SB (2008) E Doscopic Evaluatio of the Colorectum I Patie Ts Prese Ti G with Haematochezia at Korle-Bu Teachi G Hospital Accra. Ghana Med J 42(1):33-37

14. Williams MS, Kuffour G, Ekuadzi E, Yeboah M, Elduah M, Tuffour P (2013) Assessment of psychological barriers to cervical cancer screening among women in Kumasi, Ghana using a mixed methods approach. Afr Health Sci 13(4):1054-1061. https://doi. org/10.4314/ahs.v13i4.28

15. Shikarpuri S (2019) Ghana to raise daily minimum wage for 2020. https://news.bloombergtax.com/payroll/ghana-to-raisedaily-minimum-wage-for-2020. Accessed 30 July 2020

16. National Health Insurance Scheme (2020) http://www.nhis.gov. gh/benefits.aspx. Accessed 1 July 2020

Publisher's Note Springer Nature remains neutral with regard to jurisdictional claims in published maps and institutional affiliations. 